\title{
Bevacizumab for the treatment of a complicated posterior melanocytoma
}

\author{
This article was published in the following Dove Press journal: \\ Clinical Ophthalmology \\ 6 March 2015 \\ Number of times this article has been viewed
}

\section{Julio A Urrets-Zavalia* \\ Nicolas Crim* \\ Evangelina Esposito \\ Leandro Correa \\ M Eugenia Gonzalez- \\ Castellanos \\ Dana Martinez}

Department of Ophthalmology, University Clinic Reina Fabiola, Universidad Catolica de Cordoba, Cordoba, Argentina

*These authors contributed equally to this work
Correspondence: Julio A Urrets-Zavalia Department of Ophthalmology, University Clinic Reina Fabiola, Universidad Catolica de Cordoba, Oncativo 1248, Cordoba 5000, Argentina Tel +54 35I 4I4 2I50

Email julioaurrets@gmail.com
Purpose: To present a case of a complicated posterior melanocytoma that was successfully treated with intravitreal bevacizumab.

Case report: A 50-year-old Caucasian man was referred with sudden-onset metamorphopsia and decreased vision in his right eye over the course of the last 2 months. His best-corrected visual acuity was 20/80 and poorer than Jaeger 14 in the right eye, and 20/20 and Jaeger 1 in his left eye. In the right fundus, there was a melanocytic lesion occupying the inferotemporal quadrant of the optic disk, extending to the adjacent choroid inferiorly; optic nerve edema, superotemporal retinal vein dilatation, and subretinal fluid under the macula and nasal half of the posterior pole were observed, and a subretinal choroidal neovascularization complex was observed adjacent to the superotemporal margin of the optic disk, confirmed by fluorescein angiography, surrounded by a dense subretinal hemorrhage. Optical coherence tomography showed retinal edema and detachment of neurosensory retina. The patient was treated with three consecutive doses on a monthly basis of intravitreal $1.25 \mathrm{mg} / 0.05 \mathrm{~mL}$ bevacizumab. Visual acuity recovered rapidly, and at 4 months after treatment, it was 20/20 and Jaeger 1, with complete resolution of macular edema and subretinal fluid and hemorrhage. After 3 years of follow-up, best-corrected visual acuity remained stable, macular area was normal, and there was no evident optic nerve edema, retinal vein caliber and aspect were normal, and there was no significant change of the tumor. Fluorescein angiography only evidenced late staining of choroidal neovascularization scar, and optical coherence tomography showed a normal macular anatomy.

Conclusion: Intravitreal bevacizumab was effective in the treatment of choroidal neovascularization, optic nerve edema, venous dilatation, and local capillary telangiectasia, complicating an optic disk melanocytoma.

Keywords: optic disk melanocytoma, choroidal neovascularization, bevacizumab, retinal telangiectasia

\section{Introduction}

Usually unilateral, posterior melanocytoma presents clinically as an intense black or dark brown mass that may be confined to the optic disk and deeper portions of the optic nerve, but it frequently extends into the nerve fiber layer of the retina and the choroid. ${ }^{1,2}$

Although suspected to be a congenital lesion, optic disk melanocytoma is generally observed in adulthood, with a mean age of 50 years at diagnosis, and is slightly more frequent in women, with no racial predilection. ${ }^{2}$ In general, posterior melanocytoma is a stable lesion, but discrete enlargement over the course of several years may be observed in $10 \%-15 \%$ of cases. ${ }^{1,3,4}$

Primary malignant melanoma of the optic disk is extremely rare, ${ }^{5,6}$ and malignant transformation of a posterior melanocytoma has been observed in only $1 \%-2 \%$ of cases, manifesting mainly by extensive growth and severe visual loss. ${ }^{4,7}$ However, although rarely, some nonmalignant complications from an optic disk melanocytoma such as 
optic nerve edema, intraretinal and/or subretinal fluid, lipid deposits or hemorrhage, optic neuritis from ischemic tumor necrosis, and compressive central retinal vein occlusion, among others, may also produce severe visual loss. ${ }^{1}$

More recently, it has been recognized that intraretinal and/or subretinal fluid or hemorrhage in the macular area extending from a choroidal neovascularization (CNV) complex or polypoidal choroidal vasculopathy near or adjacent to an optic disk melanocytoma may also be at the origin of a severe visual loss. ${ }^{4,8,9}$

Idiopathic polypoidal choroidal vasculopathy associated with melanocytoma of the optic disk has been treated successfully with argon laser photocoagulation in one case. ${ }^{10}$ In addition, photodynamic therapy (PDT) in one case ${ }^{11}$ and intravitreous antivascular endothelial growth factor therapy in two other cases ${ }^{12,13}$ have been reported to be effective in the treatment of a complicating CNV.

We report a new case of a posterior melanocytoma associated with a CNV complex, as well as the results obtained with intravitreal bevacizumab. Also, we report for the first time the observation of epipapillary and peripapillary capillary telangiectasia in this complicated melanocytoma.

\section{Case report}

A 50-year-old Caucasian man was referred with sudden-onset and persistent metamorphopsia and severely decreased vision in his right eye over the course of the last 2 months, after a stress episode. Other than a medically controlled systemic arterial hypertension diagnosed 15 years ago, the patient was a healthy person. His best-corrected visual acuity (BCVA) was 20/80 and worse than Jaeger 14 in the right eye and 20/20 and Jaeger 1 in his left eye. Intraocular pressure was $16 \mathrm{mmHg}$ in the right eye and $12 \mathrm{mmHg}$ in the left eye, objective refraction was $-0.75 \times 170$ in both eyes, and slit-lamp biomicroscopy of both eyes was unremarkable. Right eye fundus biomicroscopy and binocular indirect ophthalmoscopy showed a slightly elevated melanocytic lesion occupying largely the inferotemporal half of the optic disk, extending temporally with feathery margins to the retina and inferiorly to the neighboring choroid. Subretinal fluid was observed under the macula and peripapillary area, extending beyond the superotemporal and inferotemporal vascular arcades, with multiple refringent fine subretinal lipid deposits spread out through the nasal half of the posterior pole. A dense accumulation of subretinal lipids was observed under the inferior margin of the optic disk, and a yelloworange, moderately protruding, subretinal mass was observed halfway between the superonasal macular area and the superotemporal optic disk margin, surrounded by a dense subretinal hemorrhage, with multiple fine subretinal folds radiating from it. In addition, a moderate optic disk edema and superior temporal and nasal retinal venous ingurgitation and tortuosity was observed (Figure 1A). The left eye was normal.

Fluorescein angiography confirmed the presumption of an active CNV complex (Figure 1B), with staining of the subretinal fluid around the optic disk in the very late angiographic phases. Time domain optical coherence tomography (Stratus ${ }^{\circledR}$; Carl Zeiss Meditec, Dublin, CA, USA) showed retinal edema and neurosensory retina detachment, with a central macular thickness of 532 microns. Systemic workup was irrelevant, and cerebral and orbital magnetic resonance imaging failed to evidence intraocular or orbital tumoral mass. The patient was treated with three consecutive doses on a monthly basis of intravitreal $1.25 \mathrm{mg} / \mathrm{mL}$ bevacizumab (Avastin ${ }^{\circledR}$; Roche Diagnostic GmbH, Manheim, Germany). Visual acuity of the right eye recovered rapidly, but not totally, after the first injection, and at 4 months after treatment, BCVA of the right eye was 20/20 and Jaeger 1. Slit-lamp biomicroscopy of the right ocular fundus showed a marked reduction of the volume of the CNV complex and no subretinal fluid, and time domain optical coherence tomography (OCT) showed a significant reduction of macular thickness (244 microns). No further treatment was performed. After 3 years of follow-up, BCVA remained stable; the right eye fundus showed a normal macular area, no evident optic nerve edema, normal retinal vein caliber and aspect, and no significant change of the tumor; the choroidal part of the tumor and the subretinal pigment dispersal were better visualized (Figure 2A). Fluorescein angiography evidenced only late staining without leakage of the CNV complex, no macular edema, and no evident capillary telangiectasia (Figure 2B). Spectral domain OCT (Cirrus $^{\circledR}$; Carl Zeiss Meditec, Dublin, CA, USA) showed a normal macular anatomy (Figure 2C), with a well-delineated domo-shaped homogeneous hyperreflective image at the level of the juxtapapillary CNV complex (Figure 2D). No ocular or systemic complications were observed during follow-up.

\section{Discussion}

Optic disk melanocytomas are usually asymptomatic, and visual loss may be the result of optic neuropathy secondary to tumor events. ${ }^{1,14}$ In our case, it seemed evident that the principal cause of metamorphopsia and visual loss was the important leakage activity of the CNV complex, as the specific treatment with an antivascular endothelial growth factor agent contributed to a rapid scarring of $\mathrm{CNV}$, with the consequent resolution of macular edema and subretinal 


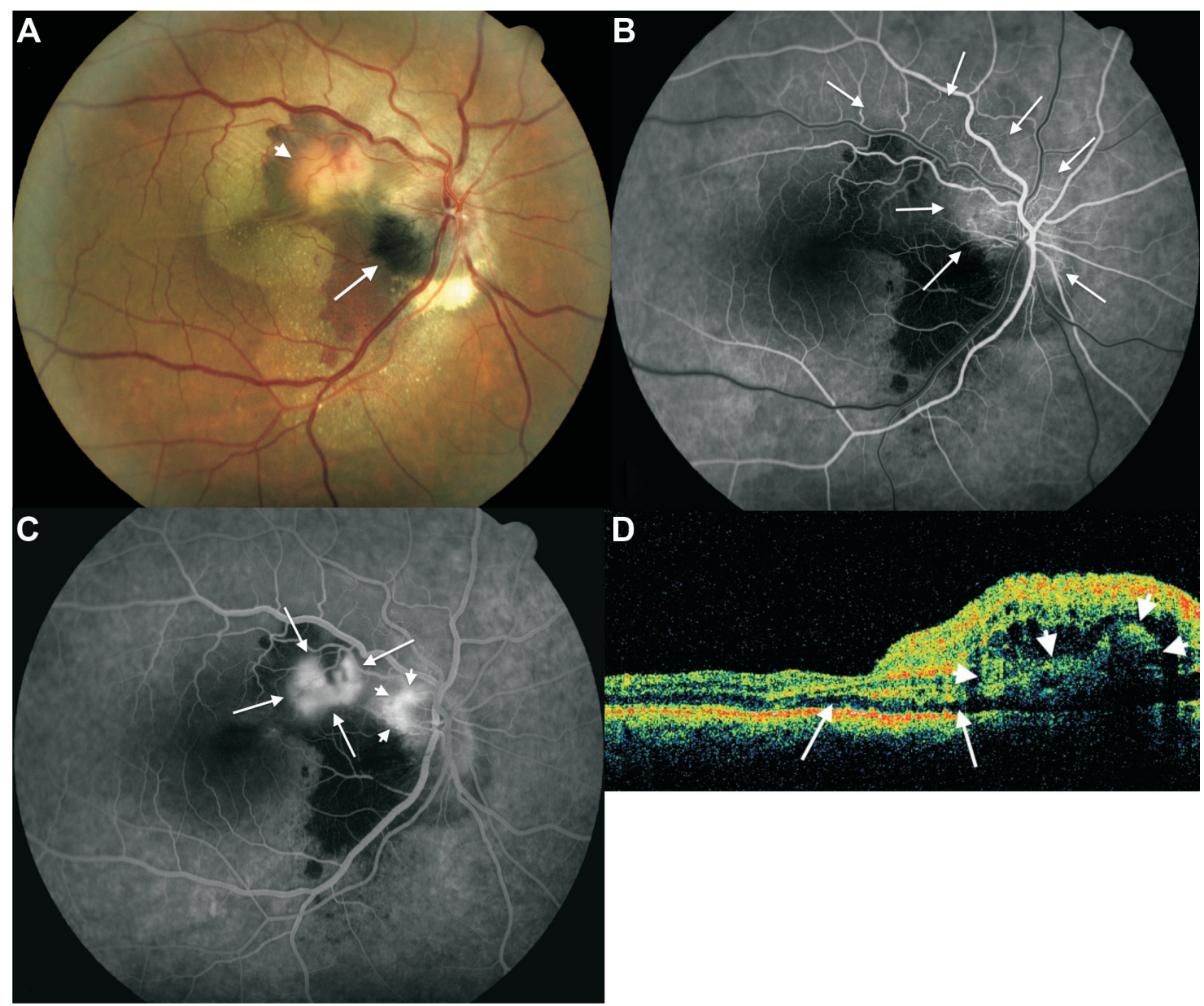

Figure I Fundus color image, fluorescein angiography and optical coherence tomography findings in patient's right eye at initial examination.

Notes: (A) Color image of the right eye fundus at first consultation, showing a deep black melanocytoma (arrow) occupying the inferotemporal half of the optic disk extending temporally to the adjacent retina (note the striated margins) and apparently to the inferior juxtapapillary choroid; subretinal choroidal neovascular complex (arrowhead) near the superotemporal margin of the optic disk, with multiple fine subretinal folds radiating from it, and two retinal folds crossing horizontally through the central macula; a myriad of fine reflective subretinal lipid deposits under the macula and nasal half of the posterior pole extending beyond the temporal vascular arcades and densely concentrated subretinal lipids at the inferior margin of the optic disk; and moderate optic disk edema and dilatation and tortuosity of superior temporal and nasal retinal veins. (B) Early arteriovenous phase fluorescein angiogram showing evident epipapillar and peripapillar retinal capillary telangiectasia, extending four-disk diameters superotemporally at both sides of the superotemporal retinal vein (arrows). (C) Venous phase fluorescein angiogram of the same eye, showing leakage from the choroidal neovascular complex (arrows) and from telangiectatic retinal capillaries of the superior juxtapapillar area (arrowheads). (D) Time domain optical coherence tomography scan passing through the fovea and choroidal neovascular complex in the same eye (arrowheads), showing shallow neurosensory detachment (arrows) and intraretinal edema in the superonasal quadrant of the macula.

fluid, and cleaning of lipid deposits and hemorrhage, with a complete and persistent restoration of far and near visual acuity and macular anatomy after a follow-up of 3 years.

The cause of subretinal choroidal neovascularization associated with an optic disk melanocytoma is unclear. It is possible that Bruch membrane could be damaged directly by tumor growth or local inflammatory events caused by the tumor itself, which could stimulate choroidal tissue to produce a neovascularization node. ${ }^{12}$ In addition to $\mathrm{CNV}$, our case presented several other pathologic events that might also be the consequence of tumor necrosis and/or enlargement, such as optic nerve edema, venous ingurgitation, and epipapillar and peripapillar retinal capillary telangiectasia, the latter observation being not previously reported. It was interesting to observe that telangiectasia and optic nerve edema became less evident rapidly after intravitreal bevacizumab treatment. It is possible that in addition to inflammatory mediators, vascular endothelial growth factor could also be involved in their pathogenesis.

As $\mathrm{CNV}$ is a rare complication of optic disk melanocytoma that has been observed in less than $1 \%$ of cases, ${ }^{4}$ there is very scant experience of its treatment, and it is confined to a handful of cases. Laser photocoagulation of the CNV complex has proven to be effective in one case associated with idiopathic polypoidal vasculopathy. ${ }^{10}$ However, the overlying and neighboring retina is destroyed by laser thermal damage, leading to an absolute scotoma in the corresponding area that may enlarge over time. PDT was effective in one case, but vision did not 


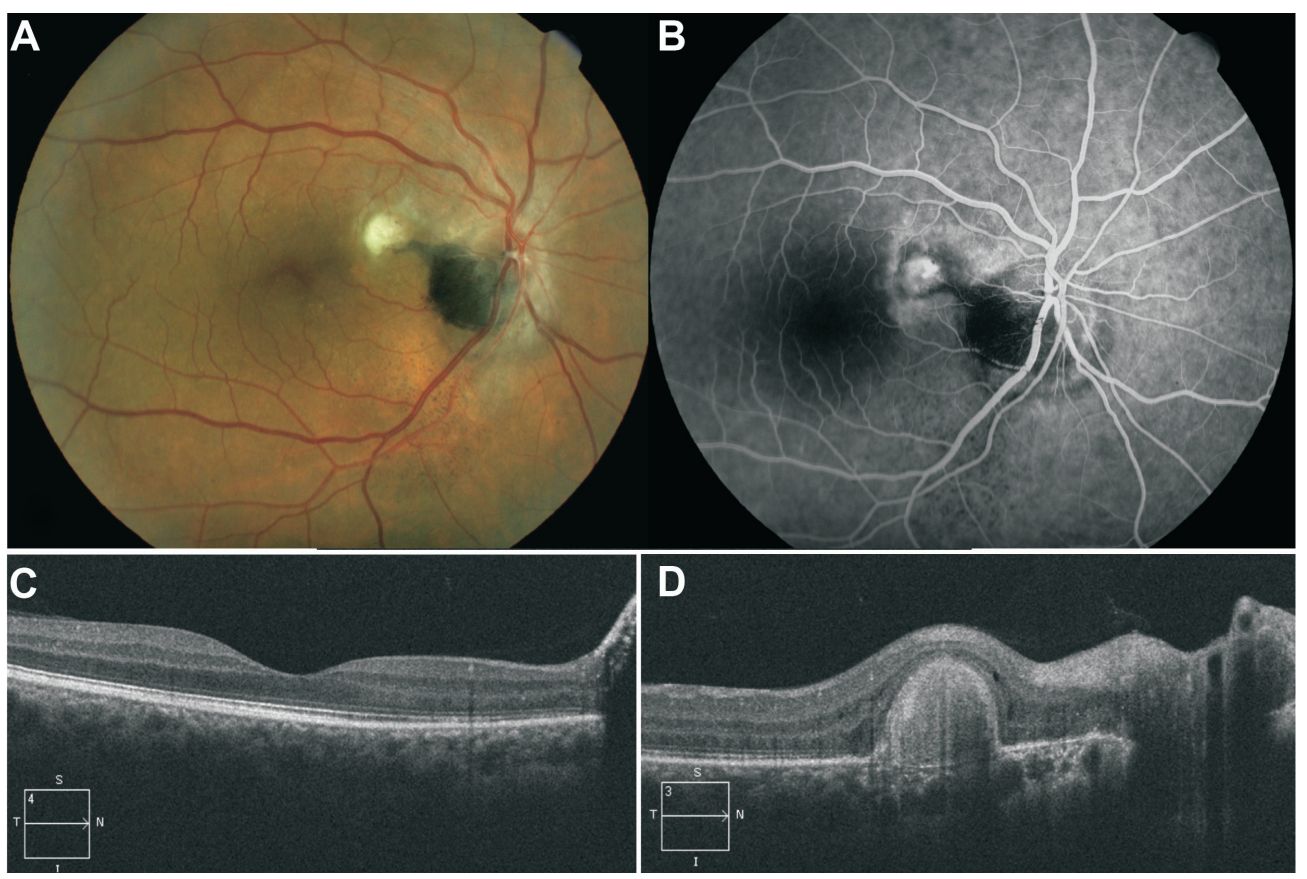

Figure 2 Post treatment fundus color image, fluorescein angiography and optical coherence tomography findings.

Notes: (A) Color image of the right eye fundus of the same patient 3 years after treatment showing complete resolution of subretinal fluid, lipid deposits and hemorrhage, and a fibrotic scar at the level of the choroidal neovascular complex; an apparent subretinal extension from the superotemporal margin of the tumor extends as a tongue toward the choroidal neovascular scar; inframacular and infrapapillar subretinal punctuate pigment dispersion is also observed, and adjacent choroidal component melanocytoma is now visible at the inferior margin of the optic disk. (B) Venous phase of fluorescein angiogram showing impregnation of choroidal neovascular scar and no capillary telangiectasia and juxtapapillar and optic disk leakage. (C) Spectral domain optical coherence tomography scan passing through the macula showing complete resolution of subretinal fluid and macular edema. (D) Spectral domain optical coherence tomography scan passing through choroidal neovascular scar.

recover completely. ${ }^{11}$ As $\mathrm{CNV}$ is always in the vicinity of the optic nerve, PDT may have a deleterious effect on its neural fibers. In our case, focal laser and PDT would have only dealt with CNV exudative complications, but not with optic nerve edema, venous dilatation, and capillary telangiectasia. Intravitreal bevacizumab has been found to be effective in two previous cases, with CNV complicating an optic disk melanocytoma with only one injection ${ }^{13}$ or with three bimonthly injections, ${ }^{12}$ both cases with a follow-up of 1 year. In our case, we obtained a very good result with three injections, with stability of vision and tumor after a follow-up of 3 years.

\section{Conclusion}

CNV seems to form part of different events that occur in the context of a complicated posterior melanocytoma. Treatment with intravitreous injections of bevacizumab was found to be very effective for complicating $\mathrm{CNV}$, as well as contributing to resolve optic disk edema, retinal venous ingurgitation, and epi/peripapillar retinal capillary telangiectasia.

\section{Author contributions}

JAU-Z performed conception and design of the work; ophthalmologic examination, treatment, and follow-up of patient; analysis and interpretation of data; writing of the manuscript and text editing; figure selection and editing; and final manuscript critical revision and correction. NC performed conception and design of the work, follow-up of patient, analysis and interpretation of data, writing of the manuscript and text editing, figure editing, and final manuscript critical revision and correction. EE performed conception and design of the work, follow-up of patient, analysis and interpretation of data, writing of the manuscript and text editing, and final manuscript critical revision and correction. LC performed follow-up of patient; acquisition, analysis, and interpretation of data; figure selection and editing; and manuscript revision and text editing. MEG-C performed follow-up of patient; acquisition, analysis, and interpretation of data; and manuscript revision and text editing. DM performed analysis and interpretation of data and manuscript revision. All authors read and approved the final manuscript and are accountable for all aspects of the work in ensuring that questions related to the accuracy or integrity of any part of the work were appropriately investigated and resolved.

\section{Disclosure}

The authors report no conflicts of interest in this work. 


\section{References}

1. Joffe L, Shields JA, Osher RH, Gass JDM. Clinical and follow-up studies of melanocytomas of the optic disc. Ophthalmology. 1979;86(6):1067-1078.

2. Shields JA, Demirci H, Mashayekhi A, Eagle RC Jr, Shields CL. Melanocytoma of the optic disk: a review. Surv Ophthalmol. 2006;51(2):93-104.

3. Gass JDM. Problems in the differential diagnosis of choroidal nevi and malignant melanomas. The XXXIII Edward Jackson Memorial Lecture. Am J Ophthalmol. 1977;83(3):299-323.

4. Shields JA, Demirci H, Mashayekhi A, Shields CL. Melanocytoma of optic disc in 115 cases: the 2004 Samuel Johnson Memorial Lecture, part 1. Ophthalmology. 2004;111(9):1739-1746.

5. Erzurum SA, Jampol LM, Territo C, O'Grady R. Primary malignant melanoma of the optic nerve simulating a melanocytoma. Arch Ophthalmol. 1992;110(5):684-686.

6. De Potter P, Shields CL, Eagle RC Jr, Shields JA, Lipkowitz JL. Malignant melanoma of the optic nerve. Arch Ophthalmol. 1996;114(5): 608-612.

7. Meyer D, Ge J, Blinder KJ, Sinard J, Xu S. Malignant transformation of an optic disk melanocytoma. Am J Ophthalmol. 1999;127(6):710-714.

8. Bartlett HM, Willoughby B, Mandava N. Polypoidal choroidal vasculopathy in a patient with melanocytoma of the optic nerve. Retina. 2001; 21(4):396-399.
9. Chalam KV, Shah GY, Shah VA, Rappaport KD. Choroidal neovascular membrane associated with melanocytoma of the optic nerve. Retina. 2006;26(6):703-704.

10. Nicolás El-Haddad M, Clement Fernández F, Pérez-Salvador E, Martín Reyes C. Vasculopatía coroidal polipoidea en paciente con melanocitoma del nervio óptico. [Polypoidal choroidal vasculopathy in a patient with a melanocytoma of the optic nerve]. Arch Soc Esp Oftalmol. 2002;77(8):455-458. Spanish.

11. Chalam KV, Gupta SK, Shah GY, Agarwal S. Successful management of melanocytoma-associated choroidal neovascularization with photodynamic therapy. Eur J Ophthalmol. 2006;16(5):776-778.

12. Kamisasanuk T, Uchino E, Sakamoto T. Choroidal neovascularization of optic disk melanocytoma treated with bevacizumab. Eur J Ophthalmol. 2012;22(3):503-505.

13. Al-Halafi AM. Successful treatment of melanocytoma associated choroidal neovascular membrane with intravitreal bevacizumab. Saudi J Ophthalmol. 2013;27(2):117-119.

14. Hajji Z, Charif Chefchaouni M, Chaoui Z, Agnaou L, Berraho A. Difficultés diagnostiques face à une tumeur papillaire pigmentée. A propos d'un cas. [Diagnostic difficulties in pigmented tumor of the optic nerve head. A case report]. J Fr Ophtalmol. 2005;28(6):614-617. French.
Clinical Ophthalmology

\section{Publish your work in this journal}

Clinical Ophthalmology is an international, peer-reviewed journal covering all subspecialties within ophthalmology. Key topics include: Optometry; Visual science; Pharmacology and drug therapy in eye diseases; Basic Sciences; Primary and Secondary eye care; Patient Safety and Quality of Care Improvements. This journal is indexed on

Submit your manuscript here: http://www.dovepress.com/clinical-ophthalmology-journal

\section{Dovepress}

PubMed Central and CAS, and is the official journal of The Society of Clinical Ophthalmology (SCO). The manuscript management system is completely online and includes a very quick and fair peer-review system, which is all easy to use. Visit http://www.dovepress.com/ testimonials.php to read real quotes from published authors. 